Canadian

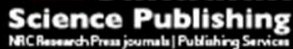

Applied Physiology, Nutrition, and Metabolism Physiologie appliquée, nutrition et métabolisme

\title{
Weight loss enhances hepatic antioxidant status in a NAFLD model induced by high fat diet
}

\begin{tabular}{|c|c|}
\hline Journal: & Applied Physiology, Nutrition, and Metabolism \\
\hline Manuscript ID & apnm-2017-0317.R1 \\
\hline Manuscript Type: & Article \\
\hline Date Submitted by the Author: & 05-Aug-2017 \\
\hline Complete List of Authors: & $\begin{array}{l}\text { Mendes, Iara; Universidade do Estado do Rio de Janeiro, Pharmacology and } \\
\text { Psychobiology } \\
\text { Matsuura, Cristiane; Universidade do Estado do Rio de Janeiro, } \\
\text { Pharmacology and Psychobiology } \\
\text { Aguila, Marcia; Universidade do Estado do Rio de Janeiro, Anatomy } \\
\text { Daleprane, Julio; State University of Rio de Janeiro, Nutrition Institute } \\
\text { Martins, Marcela; Universidade do Estado do Rio de Janeiro, Pharmacology } \\
\text { and Psychobiology } \\
\text { Mury, Wanda; Universidade do Estado do Rio de Janeiro, Pharmacology } \\
\text { and Psychobiology } \\
\text { Brunini, Tatiana; Universidade do Estado do Rio de Janeiro, Pharmacology } \\
\text { and Psychobiology }\end{array}$ \\
\hline \multicolumn{2}{|l|}{$\begin{array}{r}\text { Is the invited manuscript for } \\
\text { consideration in a Special } \\
\text { Issue? : }\end{array}$} \\
\hline Keyword: & obesity, oxidative stress, fatty liver, weight loss, diet \\
\hline
\end{tabular}




\section{Weight loss enhances hepatic antioxidant status in a NAFLD model induced by high fat} diet

Iara Karise Santos Mendes ${ }^{\mathrm{a}}$, Cristiane Matsuura ${ }^{\mathrm{a}}$, Marcia Barbosa Aguila ${ }^{\mathrm{b}}$, Julio Beltrame Daleprane $^{\mathrm{c}}$, Marcela Anjos Martins ${ }^{\mathrm{a}}$, Wanda Vianna Mury ${ }^{\mathrm{a}}$, Tatiana Marlowe Cunha Brunini ${ }^{\mathrm{a}}$

${ }^{a}$ Department of Pharmacology and Psychobiology, University of the State of Rio de Janeiro, Rio de Janeiro, Brazil.

${ }^{\mathrm{b}}$ Laboratory of Morphometry and Cardiovascular Morphology, University of the State of Rio de Janeiro, Rio de Janeiro, Brazil.

${ }^{\mathrm{c}}$ Laboratory for Studies on Interactions between Nutrition and Genetics, Department of Basic and Experimental Nutrition, University of the State of Rio de Janeiro, Rio de Janeiro, Brazil.

E-mail addresses:

Iara K Mendes, iarakarise@hotmail.com; Cristiane Matsuura, cristiane.matsuura@pq.cnpq.br; Marcia B Aguila, marciaguila@gmail.com; Julio B Daleprane, juliobd@gmail.com; Marcela A Martins, marcelaanjosm@gmail.com; Wanda V Mury, wandavianna@hotmail.com; Tatiana M C Brunini, tmcbrunini@yahoo.com.br

Corresponding author

Cristiane Matsuura, Departamento de Farmacologia e Psicobiologia, Universidade do Estado do Rio de Janeiro, Rio de Janeiro, 20551-030, Brazil, Tel.: 0055212868 8652, Fax: 0055

212868 8629, E-mail: cristiane.matsuura@pq.cnpq.br 


\section{ABSTRACT}

Purpose. Nonalcoholic fatty liver disease (NAFLD) is a benign condition that can progress to more severe liver damage in a process mediated, in part, by disturbances in redox balance. Additionally, some argue that it is set to become the main cause of end-stage liver disease in the near future. Here, we investigated whether a diet-induced weight loss is able to reverse hepatic lipid accumulation and to reduce oxidative stress in liver from C57BL/6 mice fed a high-fat (HF) diet.

Methods and results. Male C57BL/6 mice were divided into four groups: SC (standard chow, 10\% energy from fat, $16 \mathrm{wk}$ ); HF (high fat diet, 50\% energy from fat, $16 \mathrm{wk}$ ); SC-HF (SC 8 wk followed by HF 8 wk); and HF-SC (HF 8 wk followed by SC 8 wk). The HF diet during 8 (SC-HF) and 16 weeks (HF) downregulated mRNA levels and protein expression of Nrf2 and endogenous antioxidant enzymes (superoxide dismutase, catalase, glutathione peroxidase and glutathione reductase) in the liver, caused liver steatosis, affected liver function markers, increased intra-abdominal and subcutaneous adipose tissue, and induced glucose intolerance and hypercholesterolemia compared to controls (SC). Diet-induced weight loss significantly reduced the intrahepatic lipid accumulation, improved glucose tolerance, and restored both gene and protein expression of the antioxidant enzymes.

Conclusion. Our findings suggest that a dietary intervention aimed to induce weight loss may exert protective effects in NAFLD as it can reduce hepatic oxidative stress and intrahepatic lipid accumulation, which can hinder the progression of this condition to more severe states.

Keywords: fatty liver, obesity, oxidative stress, weight loss, diet 


\section{INTRODUCTION}

The rising prevalence of obesity has led to the development of non-traditional deleterious health issues, and this includes non-alcoholic fatty liver disease (NAFLD), which is now the most common liver disorder in the Western world (Bellentani 2017). NAFLD is characterized by excessive lipid deposition in the hepatocyte, which in a subset of patients evolves to an inflammatory condition with hepatocyte damage (non-alcoholic steatohepatitis, NASH) that can further progress to end-stage liver disease (Chalasani et al. 2012). In addition, NAFLD is intrinsically related to hepatic insulin resistance and abnormal glucose metabolism, but it is still debated if as a cause or as a consequence (Serviddio et al. 2013). Thus, it is imperative to improve the understanding of its causative factors and therapeutic options.

Obesity may lead to NAFLD by two major pathways: (1) increased hepatic inflow of free fatty acids; and (2) in the presence of insulin resistance, there is also an increase intrahepatic synthesis of free fatty acids by de novo lipogenesis, as well as reduction in $\beta$ oxidation and VLDL assembly, resulting in increased triglycerides accumulation (Dietrich and Hellerbrand 2014). However, it is important to highlight that free fatty acids are also capable of causing insulin resistance through serine-kinase activation (Buzzetti et al. 2016), thus perpetuating the deleterious insult. NAFLD progression to more severe states is a complex phenomenon in which different events may be occurring in parallel in a genetically susceptible individual. Increased oxidative stress is one of these multiple factors that can contribute to NASH, as it may trigger the activation of inflammatory, apoptotic and fibrotic pathyways (Buzzetti et al. 2016). Excessive lipid accumulation in the hepatocyte, as well as inflammatory cytokines released from adipose tissue or endotoxin derived from the gut, may trigger endoplasmic reticulum stress, mitochondrial dysfunction and lysosomal permeability, leading to increased reactive oxygen species (ROS) production (Feldstein and Bailey 2011). This seems to occur in a setting of reduced activity of endogenous antioxidant defences 
(Videla et al. 2004; Feng et al. 2017), even though divergent findings have been reported in both humans (Perlemuter et al. 2005) and rodents (de Assis et al. 2012). In addition, it is not known whether their expression levels are altered in NAFLD. But, regardless of the expression and/or activity of antioxidant enzymes, it has been consistently shown that oxidative stress and then damage to DNA, lipids, and proteins ensues in NAFLD. Lipid peroxidation by products are extremely reactive and may lead to activation of stellate cells and a proinflammatory response from Kupffer cells (Lee et al. 1995). Both factors, in turn, contribute to the perpetuation of the inflammatory process and fibrosis development.

To date, changes in lifestyle are considered the mainstay of the management of NAFLD. Weight loss, induced by dietary restriction or increased physical activity, usually results in a reduction in hepatic steatosis (strength 1 , level of evidence A, according to the American Gastroenterological Association, American Association for the Study of Liver Diseases, and American College of Gastroenterology position statement) (Chalasani et al. 2012). In addition, diet- or surgery- induced weight loss has been shown to reduce systemic (Razavi Zade et al. 2016) and hepatic (Bell et al. 2010) lipid peroxidation markers. To our knowledge, it is not known, however, whether diet-induced weight loss may improve liver redox state and the complications associated with an excess of intrahepatic lipid accumulation.

\section{MATERIALS AND METHODS}

\section{Animals}

The experimental protocol was approved by the Ethics Committee for the Care and Use of Experimental Animals of the State University of Rio de Janeiro (CEA/ 033/2011). Forty male C57BL/6 mice were housed in a room with controlled temperature $\left(21 \pm 1^{\circ} \mathrm{C}\right)$ and 
humidity $(60 \pm 10 \%)$, with a $12 \mathrm{~h}$ light/ dark cycle (lights on: 01:00 AM to 01:00 PM) and free access to food and water.

At three months of age, mice were randomly divided into four groups $(n=10$, each), according to the diet received: (a) standard chow (SC, 16 weeks of standard chow); (b) high fat (HF, 16 weeks of high fat diet); (c) SC-HF ( 8 weeks of SC followed by 8 weeks of HF diet); and (d) HF-SC ( 8 weeks of HF followed by 8 weeks of SC). A detailed diet description is given below.

\section{Experimental diets}

Diets were manufactured by PragSoluções (PragSolucoes, SP, Brazil) in accordance with the AIN-93M recommendations (Reeves et al. 1993). The standard chow (SC) was composed of $76 \%$ energy from carbohydrates, 14\% energy from protein, and $10 \%$ energy from lipids (total energy $15 \mathrm{KJ} / \mathrm{g}$ ). The high-fat diet (HF) was composed of $36 \%$ energy from carbohydrates, 14\% energy from protein, 50\% energy from lipids (43\% lard, $7 \%$ soybean oil) (total energy $21 \mathrm{KJ} / \mathrm{g}$ ). The mineral and vitamin contents were identical in both diets.

\section{Body mass and energy intake}

Body mass was measured every week and dietary intake was monitored daily. Food was freely allowed and fresh chow was provided daily. Food intake was determined as the difference between the amount of food supplied (g) and the amount of food left in the grid (g), divided by the number of animals per cage. Energy intake was calculated as the product of food intake $(\mathrm{g})$ and the respective energy content of the $\operatorname{diet}(\mathrm{KJ} / \mathrm{g})$.

\section{Oral glucose tolerance test}


An oral glucose tolerance test (OGTT) was conducted two days before sacrifice. Glucose $(1.0 \mathrm{~g} / \mathrm{kg})$ was administered by orogastric gavage after a six-hour fasting period. Glycemia was measured with a glucometer (Accu-Chek Performa, Roche Diagnostic, Manheim, Germany) from blood collected from the tail vein in the fasting state $(0 \mathrm{~min})$ and $15,30,60$ and 120 minutes after glucose administration.

\section{Euthanasia}

On the day before euthanasia, animals were deprived of food for 6 hours prior to being anesthetized with thiopental sodium $(0.42 \mathrm{mg} / \mathrm{g}$, i.p. $)$ and then blood samples were obtained by cardiac puncture through the right atrium. The blood was collected in tubes containing heparin, which was further centrifuged for plasma obtainment. The liver was dissected and weighed, fixed in Millonig formalin and processed for light microscopy. Liver fragments were also frozen at $-80^{\circ} \mathrm{C}$ for Western blotting, RT-PCR and enzymatic assays. The inguinal, retroperitoneal and epididymal adipose tissue were dissected and weighed to calculate the index of adiposity.

\section{Adiposity index}

The inguinal fat pad, located between the lower part of the rib cage and the mid-thigh, was considered subcutaneous fat. Retroperitoneal fat (connected to the posterior abdominal wall near the kidneys and the abdominal portion of the ureters) and epididymal fat (located in the lower part of the abdomen and connected to the epididymis) were considered the intraabdominal fat pad. The adiposity index was determined as the ratio between the sum of intraabdominal and subcutaneous adipose tissue divided by total body mass, presented as a percentage. 


\section{Biochemical measurements}

Total cholesterol and triglyceride serum levels were measured by a colorimetric assay (Bioclin, Belo Horizonte, MG, Brazil). In order to assess liver function, alanine aminotransferase serum levels were measured using an automated chemistry analyzer (Cobas $6000^{\mathrm{TM}}$, Roche, Switzerland).

\section{Gene expression of Keap1, Nrf2 and antioxidant enzymes}

The liver was homogenized under liquid nitrogen using a polytron, and total RNA was extracted using Trizol reagent (Invitrogen, Carlsbad, CA, USA). RNA concentration was determined using Nanovue (GE Life Sciences) spectroscopy, and $1 \mu \mathrm{g}$ of RNA was treated with DNAse I (Invitrogen). Synthesis of the first strand cDNA was performed using Oligo (dT) primers for mRNA and Superscript III reverse transcriptase (both from Invitrogen). Quantitative real-time PCR (qPCR) was performed using a BioRad CFX96 cycler and SYBR Green mix (Invitrogen). Primers for qPCR were designed using Primer3 online software (Table 1). Beta-actin was used as the endogenous control to normalize expression of the selected genes. The efficiency of qPCR for the target gene and endogenous control were approximately equal and were calculated through a cDNA dilution series. The PCR reactions were conducted as follows: after a pre-denaturation and polymerase activation program (4 min at $95^{\circ} \mathrm{C}$ ), forty-four cycles, each consisting of $95^{\circ} \mathrm{C}$ for $10 \mathrm{~s}$ and $60^{\circ} \mathrm{C}$ for $15 \mathrm{~s}$, were performed according to a melting curve program $\left(60\right.$ to $95^{\circ} \mathrm{C}$ with a heating rate of $\left.0.1^{\circ} \mathrm{C} / \mathrm{s}\right)$. Negative controls consisted of wells in which cDNA was substituted for deionized water. The relative expression ratio of mRNA was calculated by the $2-\Delta \Delta \mathrm{Ct}$ equation where $-\Delta \mathrm{CT}$ represents the difference between the number of cycles (CT) of the target genes and the endogenous control. 
Western Blot for superoxide dismutase (SOD), catalase, glutathione peroxidase (GPx), and glutathione reductase (GR)

Liver fragments $(100 \mathrm{mg})$ were added to a lysis buffer containing protease inhibitors, homogenized and centrifuged (4500 rpm during 20 minutes at $4{ }^{\circ} \mathrm{C}$ ). Protein concentration was then determined using the BCA protein assay kit (Thermo Scientific, Rockford, IL, USA). After denaturation, proteins were separated by electrophoresis on a polyacrylamide gel (SDS-PAGE) and transferred to a PVDF membrane. Membranes were then blotted with primary antibodies for SOD, catalase, glutathione peroxidase (GPx) and glutathione reductase (GR), followed by incubation with proper horseradish peroxidase-conjugated secondary antibody. Bands were detected by chemiluminescence (ECL Prime, Amersham, UK) using the ChemiDoc system (Bio-Rad, Hercules, CA, USA). The intensity of the bands was quantified using ImageJ software (NIH, imagej.nih.gov/ij, USA). The expression of the structural protein $\beta$-actin was used to correct the blot data. Both primary and secondary antibodies were purchased from SantaCruz Biotechnology, CA, USA.

\section{Antioxidant enzyme activity assays}

SOD, catalase and GPx activity were determined in liver homogenate by spectrophotometry (Genesys ${ }^{\mathrm{TM}} 10 \mathrm{~S}$ UV-Vis Spectrophotometer, Thermo Scientific, CA, USA). Catalase activity was measured by the rate of decrease in hydrogen peroxide concentration (Aebi 1984). SOD activity was assayed by measuring adrenochrome concentration which results from adrenaline autoxidation (Sun and Zigman 1978). GPx activity was measured by monitoring the oxidation of NADPH at $340 \mathrm{~nm}$ in the presence of hydrogen peroxide (Flohe and Gunzler 1984). The total protein content of each sample was determined by BCA protein assay kit (Thermo Scientific, Rockford, IL, USA). 


\section{Oxidative damage}

Lipid peroxidation was assessed by thiobarbituric acid reactive substances (TBARS) assay, as previously described by Draper and co-workers (1993). In brief, liver samples were mixed with $10 \%$ trichloroacetic acid and $0.67 \%$ thiobarbituric acid, followed by heating in a boiling water bath for 30 minutes. TBARS levels were determined by absorbance at $532 \mathrm{~nm}$ (Genesys $^{\mathrm{TM}}$ 10S UV-Vis Spectrophotometer, Thermo Scientific, CA, USA) using 1,1,3,3tetramethoxy propane as a standard. In addition, we measured 4-hydroxynonenal (4-HNE) and 3-nitrotyrosine (3-NT) modified proteins by Western Blot, using the same procedures described before in 'Western Blot', except for the use of primary antibody against 4-HNE (1:500, EMD Millipore, CA, USA) or 3-NT (1:1000, Santa Cruz Biotechnology, CA, USA).

\section{Liver structure}

The liver was weighed on a digital analytical balance to an accuracy of $0.001 \mathrm{~g}$ (Shimadzu, Japan) and its volume was determined by the method of Scherle (1970). Livers were fixed in Millonig formalin, processed by routine histological procedures, and embedded in Paraplast Plus (Sigma-Aldrich, St Louis, MO, USA). The material was sectioned at $5 \mu \mathrm{m}$ and stained with hematoxylin-eosin. A piece of each of the five lobes of each animal was selected for processing, in order to assure that the organ was analysed as a whole. Digital images were acquired at random using a Leica DMRBE microscope (Leica Microsystems, Wetzlar, Germany) and an Infinity 1-5c camera (Lumenera, Ottawa, ON, Canada). Five random microscopic fields per animal were analyzed. A test system with 36 points (PT) was produced by the web-based program STEPanizer (www.stepanizer.com) and superimposed on photomicrographs. We estimated the volume density of hepatic steatosis ( $\mathrm{Vv}$ [steatosis]) as: $\mathrm{Vv}$ [steatosis]: $=\mathrm{Pp}$ [steatosis] / Pt, where $\mathrm{Pp}$ is the number of points counted from the test system in the intra-hepatocyte (Tschanz et al. 2011). 


\section{Statistical analysis}

Data are shown as mean and S.E.M and they were tested for normality and homogeneity of variances. Statistical differences were assessed by a one-way analysis of variance (ANOVA) followed by Holm Sidak post-test. All analyses were performed using GraphPad Prism version 6.00 for Windows (GraphPad Software, CA, USA). $P$ values less than or equal to 0.05 were accepted as statistically significant.

\section{RESULTS}

\section{Body composition and energy intake}

Energy intake depended on the type of diet given to the animals, and, as such, it was higher when animals were fed the HF diet. The mean daily energy intake during the first half of the experimental period was significantly higher in HF and HF-SC groups (SC, $46.2 \pm 2.6$; $\mathrm{HF}, 67.0 \pm 2.4$; SC-HF, $49.6 \pm 2.5 ; \mathrm{HF}-\mathrm{SC}, 56.5 \pm 3.8 \mathrm{KJ} / \mathrm{animal} / \mathrm{day})$. And, in the second half of the experimental period, in HF and SC-HF groups (SC, 50.6 \pm 1.7 ; HF, 66.1 \pm 1.7 ; SC$\mathrm{HF}, 60.2 \pm 2.5 ; \mathrm{HF}-\mathrm{SC}, 43.1 \pm 2.5 \mathrm{KJ} /$ animal/day).

Figure 1 shows the body mass evolution during the whole experimental period. Due to the higher energy density of the diets, animals from both HF and HF-SC groups presented a continuous increase in body mass during the first eight weeks compared to SC and SC-HF groups. From week 8, there was a significant decrease in body mass in HF-SC group due to diet exchange, and at the end of experiment, body mass did not differ between HF-SC and SC groups. On the other side, animals from SC-HF group showed an increase in body mass after week 8 , and at the end of experiments, body mass was significantly higher than SC and HFSC, but significantly lower than HF. 
In addition to increased body mass, mice from HF and SC-HF groups presented significantly higher adiposity index, as well as absolute amounts of visceral and subcutaneous fat pads (Table 2).

\section{Biochemical analysis}

At the end of the study, fasting glucose levels of the HF group were significantly higher than the other groups. Moreover, mice from HF group presented reduced glucose tolerance, assessed by AUC calculation following an OGTT, compared to SC, SC-HF and HF-SC. Mice from the HF and SC-HF groups had higher total cholesterol levels than the SC and HF-SC groups. In contrast, triglyceride serum levels did not differ among groups (Table 3).

In addition, serum levels of alanine aminotransferase, a marker of liver function, was significantly lower in HF-SC compared to HF (Table 3).

\section{Biomarkers of oxidative stress in liver}

Gene expression of Nrf2 was significantly reduced by prolonged HF diet, and upregulated after weight loss. Weight loss restored both gene (Figure 2) and protein expression (Figure 3) of the antioxidant enzymes SOD, catalase, GPx and glutathione reductase in liver homogenate. In addition, weight loss significantly reduced hepatic lipid peroxidation, as evidenced by reducing MDA levels in both SC and HF-SC groups compared to HF and SC-HF groups. However, 4-HNE and 3-NT protein adducts levels did not differ among groups (Figure 4). No significant difference was observed in the activity of SOD, catalase and GPx among groups (Figure 5).

\section{Hepatic steatosis}


All groups exhibited some degree of hepatic steatosis, as shown in Figure 5. Compared to SC group, steatosis level was $418 \%$ higher in HF group, $268 \%$ in SC-HF group, and $188 \%$ in HF-SC group (Figure 6).

\section{DISCUSSION}

The main findings of the present study were that a dietary intervention aimed to induce weight loss resulted in a significant reduction in hepatic steatosis and in an upregulation of both mRNA and protein expression of the main intracellular antioxidant enzymes. These results are important as they can hinder the progression to more severe liver damage.

Oxidative stress has been postulated to be a central component in the deleterious progression of hepatic steatosis to steatohepatitis and, in some cases, to cirrhosis and liver failure (Spahis et al. 2017). Oxidative stress and damage occur in a setting of increased ROS production, reduced buffer capacity of antioxidant enzymes, or both (Halliwell 2011). Here, we demonstrated that obesity induced intrahepatic lipid oxidative damage and that weight loss was able to reduce it to levels similar to controls. This is a relevant finding since lipid peroxidation products, such as malondialdehyde, are extremely reactive and may activate hepatic stellate cells (Lee et al. 1995). When activated, these cells are the main source of collagen type I, which, in turn, is a major contributor to hepatic fibrosis (Friedman and Arthur 1989). Here, there was a significant reduction in hepatic lipid peroxidation after weight loss, and we believe that this finding may result from two main factors. Firstly, weight loss reversed the downregulation in gene and protein expression of the main intracellular antioxidant enzymes, namely SOD, catalase, GPx and glutathione reductase. These enzymes are important components of endogenous antioxidant defense and, to our knowledge, this is the first evidence demonstrating that their expression is reduced in NAFLD, and that their 
expression is restored after weight loss. Of note, there was no significant change in the activity of these enzymes. Divergent findings are reported in the literature regarding the activity of antioxidant enzymes in NAFLD, with some authors demonstrating a decrease, others no change or an increase in the activity of these enzymes (Videla et al. 2004; Perlemuter et al. 2005; de Assis et al. 2012; Feng et al. 2017). The differences might be due to the type and duration of the diet given, as well as the clinical condition of the patient. Therefore, here, changes in redox state were mostly due to the amount of antioxidant enzymes than due to their activity.

NAFLD, in all its spectrum of histological changes - from simple steatosis to cirrhosis, seems to be a particular case that does not meet the expected physiological regulatory response. In a setting of increased levels of pro-oxidants, it would be expected an upward adaptation of the antioxidant defense system, and vice versa. NAFLD and NASH are associated not only with an increase in ROS production, but also with a reduction in the activity of the main intracellular antioxidant enzymes, as well as coenzyme Q10 and glutathione levels (Rolo et al. 2012). For instance, gene expression of SOD, glutathione peroxidase and catalase is significantly decreased in liver from patients with cirrhosis secondary to NASH, but not secondary to hepatitis $\mathrm{C}$ infection or primary biliary cirrhosis (Sreekumar et al. 2003). The mechanisms beyond this "inadequate" adaptation are still unknown. One possible explanation may be related to the activation of nuclear factor erythroid 2-related factor $2(\mathrm{Nrf} 2)$, one of the most important transcription factor for the induction of many antioxidant enzymes, including SOD, glutathione peroxidase and catalase (Menegon et al. 2016). At least in obese animals fed a high fat diet, liver mRNA levels of Nrf2 and its downstream targets are reduced (Vomhof-Dekrey and Picklo 2012), which is in accordance with our findings. One important factor is that diet induced suppression of Nrf2 is reversed with a reduction in saturated fat ingestion and weight loss, as we showed in the 
present study. Increased activation of Nrf2 per se might contribute to the reduction of hepatic steatosis. Beyond its important role in the antioxidant system, Nrf2 possess regulatory function on metabolism (Vomhof-Dekrey and Picklo 2012). It has been demonstrated to decrease lipid synthesis as well to increase energy metabolism, resulting in a diminished hepatic triglycerides storage.

Secondly, it is possible that the dietary intervention resulted in a reduction in ROS production due to reduced intake of saturated fatty acids per se and to reduced total fat and energy intake leading to both weight loss and subsequent intrahepatic lipid accumulation. Saturated fatty acids as well as liver steatosis alters intracellular redox state, promoting uncoupling between glycolysis and TCA cycle, and the formation of reactive oxygen intermediates (Serviddio et al. 2013). So, weight loss reduced oxidative stress by diminishing ROS production as well as increasing endogenous antioxidant defenses.

As expected, albeit not less important, weight loss improved glucose tolerance. Indeed, weight loss is considered one of the most effective intervention to reduce both NAFLD and hepatic insulin resistance (Petersen et al. 2005), which has been confirmed in the present study. To date, there are many hypotheses trying to explain the link between these two entities. One hypothesis states that excess intrahepatic lipid accumulation leads to increase diacylglycerol content, which, in turn, translocates PKC isoform $\varepsilon$ to the membrane where it binds and inhibits the insulin receptor (Perry et al. 2014). A second hypothesis includes oxidative stress as a key mediator. As discussed before, oxidative stress is associated with the deleterious morphological liver changes caused by NAFLD. In addition, increased ROS production by a dysfunctional mitochondria and NADPH oxidase seems to precede insulin resistance development (Anderson et al. 2009) possibly via redox modifications of phosphatases and kinases involved in insulin signaling, such as p38 MAPK (Besse-Patin and Estall 2014). Once again, the normalization of both protein and mRNA expression of the main 
antioxidant enzymes in the liver caused by weight loss might have exerted beneficial effects for the prevention of insulin resistance and diabetes mellitus associated with obesity.

In conclusion, our findings emphasize the importance of body mass loss by dietary intervention and provide a framework for understanding the possible mechanistic processes by which NAFLD is developed.

\section{Acknowledgements}

We thank Prof. Carlos Alberto Mandarim-de-Lacerda who provided insight and expertise that greatly improved the manuscript, as well as the Brazilian funding agencies FAPERJ and CNPq.

\section{Conflict of interest}

We declare that we have no conflict of interest. 


\section{REFERENCES}

Aebi, H. 1984. Catalase in vitro. Methods Enzymol. 105: 121-126.

Anderson, E.J., Lustig, M.E., Boyle, K.E., Woodlief, T.L., Kane, D.A., Lin, C.T., Price, J.W., 3rd, Kang, L., Rabinovitch, P.S., Szeto, H.H., Houmard, J.A., Cortright, R.N., Wasserman, D.H., and Neufer, P.D. 2009. Mitochondrial H2O2 emission and cellular redox state link excess fat intake to insulin resistance in both rodents and humans. J. Clin. Invest. 119(3): 573-581. doi: 10.1172/JCI37048.

Bell, L.N., Temm, C.J., Saxena, R., Vuppalanchi, R., Schauer, P., Rabinovitz, M., Krasinskas, A., Chalasani, N., and Mattar, S.G. 2010. Bariatric surgery-induced weight loss reduces hepatic lipid peroxidation levels and affects hepatic cytochrome P-450 protein content. Ann. Surg. 251(6): 1041-1048. doi: 10.1097/SLA.0b013e3181dbb572.

Bellentani, S. 2017. The epidemiology of non-alcoholic fatty liver disease. Liver Int. 37 (Suppl 1): 81-84. doi: 10.1111/liv.13299.

Besse-Patin, A. and Estall, J.L. 2014. An Intimate Relationship between ROS and Insulin Signalling: Implications for Antioxidant Treatment of Fatty Liver Disease. Int. J. Cell Biol. 2014: 519153. doi: $10.1155 / 2014 / 519153$.

Buzzetti, E., Pinzani, M., and Tsochatzis, E.A. 2016. The multiple-hit pathogenesis of nonalcoholic fatty liver disease (NAFLD). Metabolism, 65(8): 1038-1048. doi: 10.1016/j.metabol.2015.12.012.

Chalasani, N., Younossi, Z., Lavine, J.E., Diehl, A.M., Brunt, E.M., Cusi, K., Charlton, M., and Sanyal, A.J. 2012. The diagnosis and management of non-alcoholic fatty liver disease: practice Guideline by the American Association for the Study of Liver Diseases, American College of Gastroenterology, and the American Gastroenterological Association. Hepatology, 55(6): 2005-2023. doi: 10.1002/hep.25762.

de Assis, A.M., Rech, A., Longoni, A., Rotta, L.N., Denardin, C.C., Pasquali, M.A., Souza, 
D.O., Perry, M.L., and Moreira, J.C. 2012. Omega3-Polyunsaturated fatty acids prevent lipoperoxidation, modulate antioxidant enzymes, and reduce lipid content but do not alter glycogen metabolism in the livers of diabetic rats fed on a high fat thermolyzed diet. Mol. Cell. Biochem. 361(1-2): 151-160. doi: 10.1007/s11010-011-1099-4.

Dietrich, P. and Hellerbrand, C. 2014. Non-alcoholic fatty liver disease, obesity and the metabolic syndrome. Best Pract. Res. Clin. Gastroenterol. 28(4): 637-653. doi: 10.1016/j.bpg.2014.07.008.

Draper, H.H., Squires, E.J., Mahmoodi, H., Wu, J., Agarwal, S., and Hadley, M. 1993. A comparative evaluation of thiobarbituric acid methods for the determination of malondialdehyde in biological materials. Free Radic. Biol. Med. 15(4): 353-363.

Feldstein, A.E. and Bailey, S.M. 2011. Emerging role of redox dysregulation in alcoholic and nonalcoholic fatty liver disease. Antioxid. Redox. Signal. 15(2): 421-424. doi: 10.1089/ars.2011.3897.

Feng, X., Yu, W., Li, X., Zhou, F., Zhang, W., Shen, Q., Li, J., Zhang, C., and Shen, P. 2017. Apigenin, a modulator of PPARgamma, attenuates HFD-induced NAFLD by regulating hepatocyte lipid metabolism and oxidative stress via Nrf2 activation. Biochem. Pharmacol. 136: 136-149. doi: 10.1016/j.bcp.2017.04.014.

Flohe, L. and Gunzler, W.A. 1984. Assays of glutathione peroxidase. Methods Enzymol. 105:114-121.

Friedman, S.L. and Arthur, M.J. 1989. Activation of cultured rat hepatic lipocytes by Kupffer cell conditioned medium. Direct enhancement of matrix synthesis and stimulation of cell proliferation via induction of platelet-derived growth factor receptors. J. Clin. Invest. 84(6): 1780-1785. doi: 10.1172/JCI114362.

Halliwell, B. 2011. Free radicals and antioxidants - quo vadis? Trends Pharmacol. Sci. 32(3): 125-130. doi: 10.1016/j.tips.2010.12.002. 
Lee, K.S., Buck, M., Houglum, K., and Chojkier, M. 1995. Activation of hepatic stellate cells by TGF alpha and collagen type I is mediated by oxidative stress through c-myb expression. J. Clin. Invest. 96(5): 2461-2468. doi: 10.1172/JCI118304.

Menegon, S., Columbano, A., and Giordano, S. 2016. The Dual Roles of NRF2 in Cancer. Trends Mol. Med. 22(7): 578-593. doi: 10.1016/j.molmed.2016.05.002.

Perlemuter, G., Davit-Spraul, A., Cosson, C., Conti, M., Bigorgne, A., Paradis, V., Corre, M.P., Prat, L., Kuoch, V., Basdevant, A., Pelletier, G., Oppert, J.M., and Buffet, C. 2005. Increase in liver antioxidant enzyme activities in non-alcoholic fatty liver disease. Liver Int. 25(5): 946-953. doi: 10.1111/j.1478-3231.2005.01126.x.

Perry, R.J., Samuel, V.T., Petersen, K.F., and Shulman, G.I. 2014. The role of hepatic lipids in hepatic insulin resistance and type 2 diabetes. Nature, 510(7503): 84-91. doi: 10.1038/nature13478.

Petersen, K.F., Dufour, S., Befroy, D., Lehrke, M., Hendler, R.E., and Shulman, G.I. 2005. Reversal of nonalcoholic hepatic steatosis, hepatic insulin resistance, and hyperglycemia by moderate weight reduction in patients with type 2 diabetes. Diabetes, 54(3): 603-608.

Razavi Zade, M., Telkabadi, M.H., Bahmani, F., Salehi, B., Farshbaf, S., and Asemi, Z. 2016. The effects of DASH diet on weight loss and metabolic status in adults with non-alcoholic fatty liver disease: a randomized clinical trial. Liver Int. 36(4): 563-571. doi: 10.1111/liv.12990.

Reeves, P.G., Nielsen, F.H., and Fahey, G.C., Jr. 1993. AIN-93 purified diets for laboratory rodents: final report of the American Institute of Nutrition ad hoc writing committee on the reformulation of the AIN-76A rodent diet. J. Nutr. 123(11): 1939-1951.

Rolo, A.P., Teodoro, J.S., and Palmeira, C.M. 2012. Role of oxidative stress in the pathogenesis of nonalcoholic steatohepatitis. Free Radic. Biol. Med. 52(1): 59-69. doi: 10.1016/j.freeradbiomed.2011.10.003. 
Scherle, W. 1970. A simple method for volumetry of organs in quantitative stereology. Mikroskopie, 26(1): 57-60.

Serviddio, G., Bellanti, F., and Vendemiale, G. 2013. Free radical biology for medicine: learning from nonalcoholic fatty liver disease. Free Radic. Biol. Med. 65: 952-968. doi: 10.1016/j.freeradbiomed.2013.08.174.

Spahis, S., Delvin, E., Borys, J.M., and Levy, E. 2017. Oxidative Stress as a Critical Factor in Nonalcoholic Fatty Liver Disease Pathogenesis. Antioxid. Redox. Signal. 26(10): 519-541. doi: 10.1089/ars.2016.6776.

Sreekumar, R., Rosado, B., Rasmussen, D., and Charlton, M. 2003. Hepatic gene expression in histologically progressive nonalcoholic steatohepatitis. Hepatology, 38(1): 244-251. doi: 10.1053/jhep.2003.50290.

Sun, M. and Zigman, S. 1978. An improved spectrophotometric assay for superoxide dismutase based on epinephrine autoxidation. Anal. Biochem. 90(1): 81-89.

Tschanz, S.A., Burri, P.H., and Weibel, E.R. 2011. A simple tool for stereological assessment of digital images: the STEPanizer. J. Microsc. 243(1): 47-59. doi: 10.1111/j.13652818.2010.03481.x.

Videla, L.A., Rodrigo, R., Orellana, M., Fernandez, V., Tapia, G., Quinones, L., Varela, N., Contreras, J., Lazarte, R., Csendes, A., Rojas, J., Maluenda, F., Burdiles, P., Diaz, J.C., Smok, G., Thielemann, L., and Poniachik, J. 2004. Oxidative stress-related parameters in the liver of non-alcoholic fatty liver disease patients. Clin. Sci. (Lond.) 106(3): 261-268. doi: $10.1042 / C S 20030285$.

Vomhof-Dekrey, E.E. and Picklo, M.J., Sr. 2012. The Nrf2-antioxidant response element pathway: a target for regulating energy metabolism. J. Nutr. Biochem. 23(10): 1201-1206. doi: 10.1016/j.jnutbio.2012.03.005. 
Table 1. Sequence of primers used in quantitative real time RT-PCR.

\begin{tabular}{lll}
\hline Target gene & $\mathbf{5} \mathbf{- 3}^{\prime}$ & Primers \\
\hline \multirow{2}{*}{$\beta$-actin } & FW & CTCCGGCATGTGCAA \\
& RV & CCCACCATCACACCCT \\
\multirow{2}{*}{ SOD } & FW & AACCATCCACTTCGAGCAGA \\
& RV & GGTCTCCAACATGCCTCTCT \\
Catalase & FW & ACATGGTCTGGGACTTCTGG \\
& RV & CAAGTTTTGATGCCCTGGT \\
GPx & FW & GTCCACCGTGTATGCCTTCT \\
& RV & TCTGCAGATCGTTCATCTCG \\
GR & FW & CACGACCATGATTCCAGATG \\
& RV & CAGCATAGACGCCTTTGACA \\
Keap1 & FW & GGACGGCAACACTGATTC \\
& RV & TCGTCTGATCTGGCTCATA \\
Nrf2 & FW & CACATCCAGACAGACACCAGT \\
& RV & CTACAAATGGGAATGTCTCTGC \\
\hline
\end{tabular}

GPx, glutathione peroxidase; GR, glutathione reductase; Keap1, Kelch-like ECH-associated protein 1; Nrf2, nuclear factor, erythroid derived 2, like 2; SOD, superoxide dismutase 
Table 2. Adipose tissue distribution and adiposity index in the different experimental groups.

\begin{tabular}{lllll}
\hline Variables & SC & HF & SC-HF & HF-SC \\
\hline Intra-abdominal fat (g) & $0.9 \pm 0.1$ & $3.0 \pm 0.2^{\mathbf{a}}$ & $2.6 \pm 0.2^{\mathbf{a}, \mathbf{b}}$ & $0.9 \pm 0.1^{\mathbf{b , c}}$ \\
Subcutaneous fat (g) & $0.30 \pm 0.05$ & $1.71 \pm 0.09^{\mathbf{a}}$ & $0.87 \pm 0.07^{\mathbf{a}, \mathbf{b}}$ & $0.34 \pm 0.03^{\mathbf{b , c}}$ \\
Adiposity index (\%) & $3.1 \pm 0.3$ & $6.9 \pm 0.4^{\mathbf{a}}$ & $7.2 \pm 0.4^{\mathbf{a}}$ & $3.2 \pm 0.2^{\mathbf{b , c}}$ \\
\hline
\end{tabular}

Values expressed as mean \pm SEM; SC, standard chow; HF, high-fat diet. Significant differences are represented by letters $(\mathrm{p} \leq 0.05)$ : [a] $v s$. SC group, [b] vs. HF group, [c] $v s$. SC-HF. 
Table 3. Biochemical analysis in the different experimental groups.

\begin{tabular}{ccccc}
\hline Variables & SC & HF & SC-HF & HF-SC \\
\hline Glucose (mmol/L) & $9.0 \pm 0.7$ & $15.6 \pm 0.5^{\mathrm{a}}$ & $11.2 \pm 0.5$ & $11.6 \pm 3.2$ \\
OGTT (a.u.c.) & $1104 \pm 36$ & $1481 \pm 112^{\mathrm{a}}$ & $1095 \pm 28^{\mathrm{b}}$ & $956 \pm 10^{\mathrm{b}}$ \\
Total cholesterol (mmol/L) & $3.5 \pm 0.2$ & $5.8 \pm 0.2^{\mathrm{a}}$ & $4.6 \pm 0.1^{\mathrm{a}, \mathrm{b}}$ & $3.4 \pm 0.3^{\mathrm{b}, \mathrm{c}}$ \\
Triglycerides (mmol/L) & $0.54 \pm 0.07$ & $0.62 \pm 0.02^{\mathrm{b}}$ & $0.53 \pm 0.10$ & $0.67 \pm 0.04$ \\
ALT (U/L) & $11 \pm 2$ & $49 \pm 10^{\mathrm{a}}$ & $18 \pm 4^{\mathrm{b}}$ & $13 \pm 4^{\mathrm{b}}$ \\
\hline
\end{tabular}

Values expressed as mean \pm SEM; SC, standard chow; HF, high-fat diet. OGTT, oral glucose tolerance test; ALT, alanine aminotransferase; a.u.c, area under the curve. Significant differences are represented by letters $(\mathrm{p} \leq 0.05)$ : [a] $v s$. SC group, [b] $v s$. HF group, [c] $v s$. SC-HF. 


\section{FIGURE CAPTIONS}

Figure 1. Body mass evolution during the experimental period. Dashed line indicates diet exchange. Data are presented as mean \pm SEM. SC, standard chow; HF, high-fat. Significant differences are represented by letters $(\mathrm{p} \leq 0.05)$ : [a] vs. SC group, [b] vs. HF group, [c] vs. SC-HF group.

Figure 2. mRNA expression of Keap1, Nrf2, and the antioxidant enzymes superoxide dismutase, catalase, glutathione peroxidase, and glutathione reductase in liver. Results are normalized by $\beta$-actin, which was used as loading control. Data are shown as mean $\pm \mathrm{SEM}$. Significant differences are represented by letters $(\mathrm{p} \leq 0.05)$ : [a] $v s . \mathrm{SC},[\mathrm{b}] v s . \mathrm{HF}$, [c] $v s . \mathrm{SC}-$ HF. HF, high fat; SC, standard chow.

Figure 3. Protein levels of the antioxidant enzymes superoxide dismutase (A), catalase (B), glutathione peroxidase (C), and glutathione reductase (D) in liver. Results are normalized by $\beta$-actin, which was used as loading control. Data are shown as mean \pm SEM. Significant differences are represented by letters $(\mathrm{p} \leq 0.05)$ : [a] vs. SC, [b] vs. HF, [c] vs. SC-HF. HF, high fat; SC, standard chow.

Figure 4. Oxidative stress biomarkers in liver homogenate. (A) malondialdehyde, (B) 4hydroxynonenal protein adducts, and (C) 3-nitrotyrosine protein adducts. Data are shown as mean \pm SEM. Significant differences are represented by letters $(\mathrm{p} \leq 0.05)$ : $[\mathrm{a}] v s . \mathrm{SC},[\mathrm{b}] v s$. HF, [c] vs. SC-HF. HF, high fat; SC, standard chow.

Figure 5. Activity of the antioxidant enzymes (A) superoxide dismutase, (B) catalase, and (C) glutathione peroxidase in liver homogenate. Data are shown as mean \pm SEM. Significant 
differences are represented by letters $(\mathrm{p} \leq 0.05)$ : [a] vs. SC, [b] vs. HF, [c] vs. SC-HF. HF, high fat; SC, standard chow.

Figure 6. Liver steatosis. (A) bar graph of the volume density of the liver steatosis (mean \pm SEM). Photomicrographs of liver tissue stained with hematoxylin-eosin in (B) SC group, (C), HF group, (D) SC-HF group, and (E) HF-HF group (bar $=50 \mu \mathrm{m}$, same magnification in all photomicrographs). Significant differences are represented by letters $(\mathrm{p} \leq 0.05)$ : [a] vs. SC, [b] vs. HF, [c] vs. SC-HF. HF, high fat; SC, standard chow. 


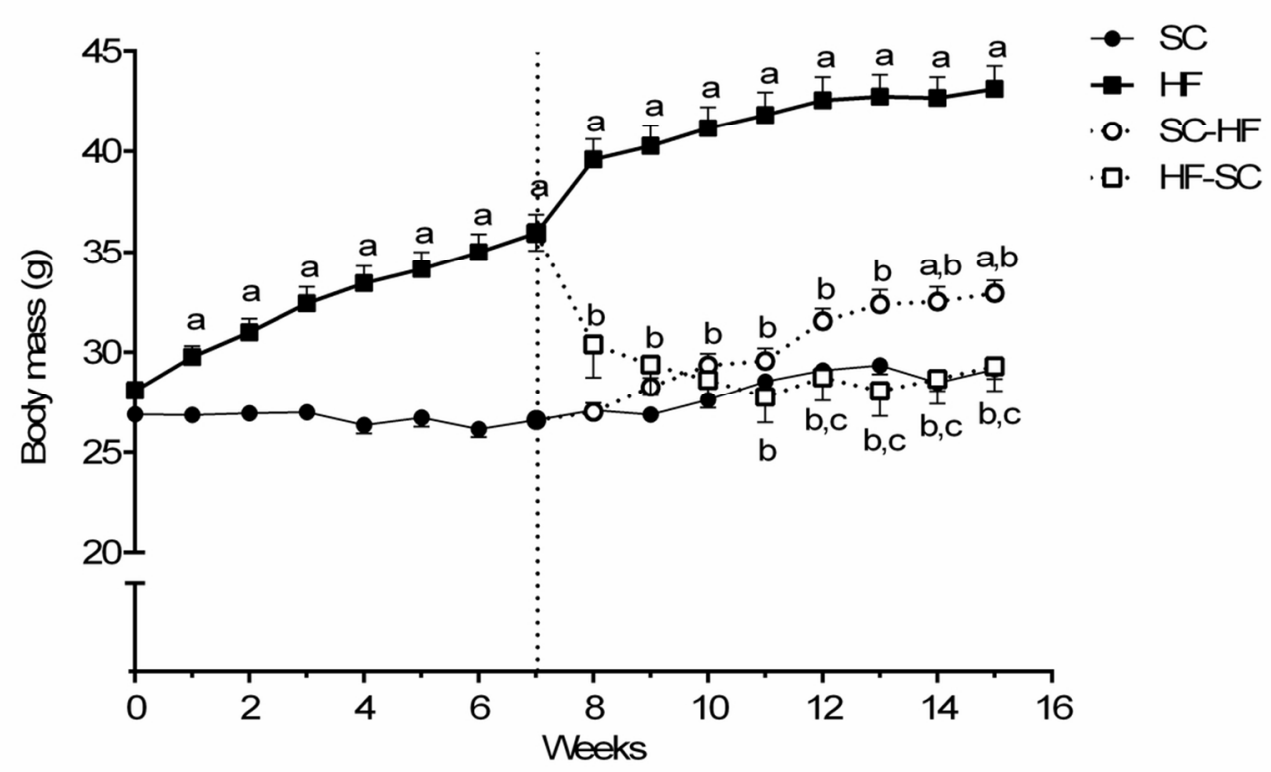

Figure 1. Body mass evolution during the experimental period. Dashed line indicates diet exchange. Data are presented as mean \pm SEM. SC, standard chow; HF, high-fat. Significant differences are represented by letters $(p \leq 0.05)$ : $[a]$ vs. SC group, [b] vs. HF group, [c] vs. SC-HF group.

$101 \times 62 \mathrm{~mm}(300 \times 300 \mathrm{DPI})$ 


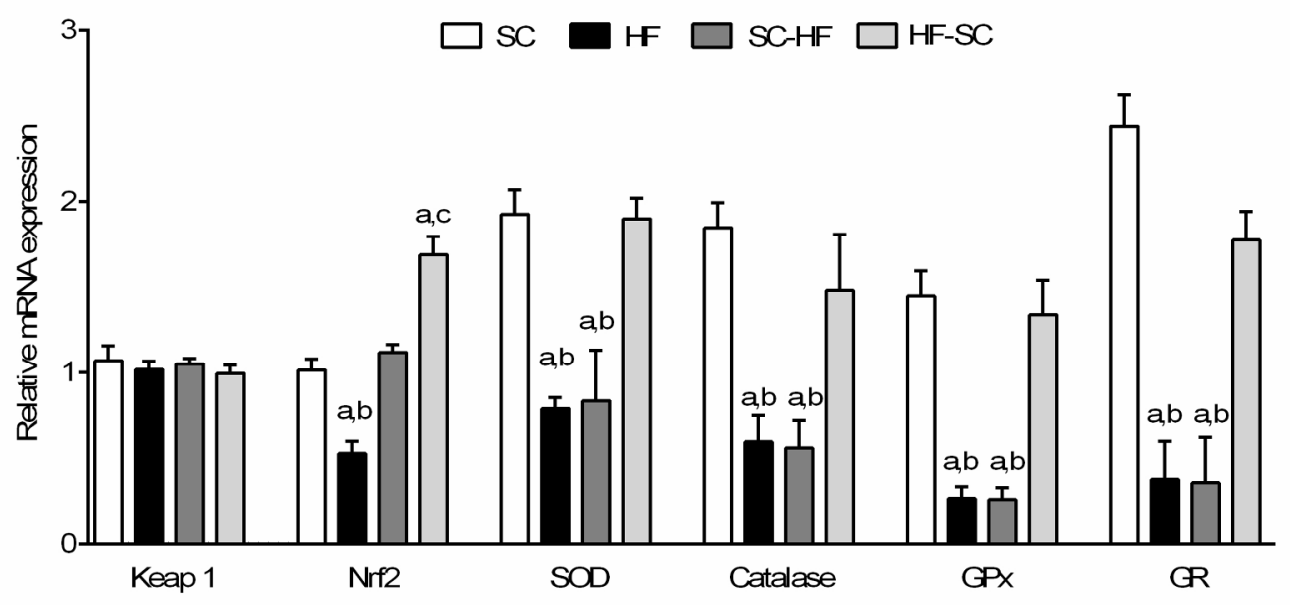

Figure 2. mRNA expression of Keap1, Nrf2, and the antioxidant enzymes superoxide dismutase, catalase, glutathione peroxidase, and glutathione reductase in liver. Results are normalized by $\beta$-actin, which was used as loading control. Data are shown as mean \pm SEM. Significant differences are represented by letters $(\mathrm{p} \leq 0.05)$ : [a] vs. SC, [b] vs. HF, [c] vs. SC-HF. HF, high fat; SC, standard chow.

$198 \times 96 \mathrm{~mm}(300 \times 300 \mathrm{DPI})$ 

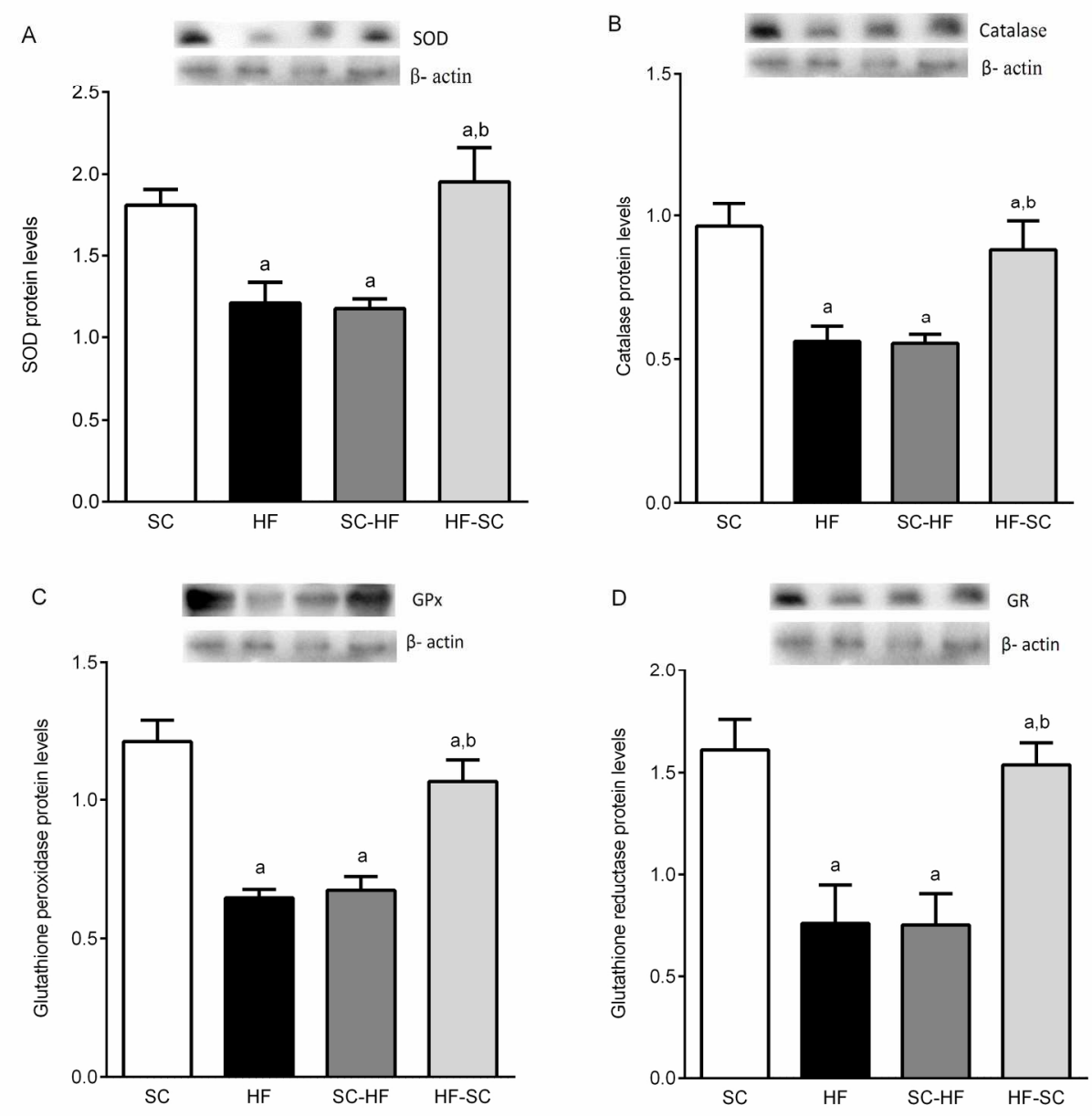

Figure 3. Protein levels of the antioxidant enzymes superoxide dismutase (A), catalase (B), glutathione peroxidase (C), and glutathione reductase (D) in liver. Results are normalized by $\beta$-actin, which was used as loading control. Data are shown as mean \pm SEM. Significant differences are represented by letters $(p \leq$ 0.05): [a] vs. SC, [b] vs. HF, [c] vs. SC-HF. HF, high fat; SC, standard chow.

$$
189 \times 187 \mathrm{~mm} \text { ( } 300 \times 300 \text { DPI) }
$$



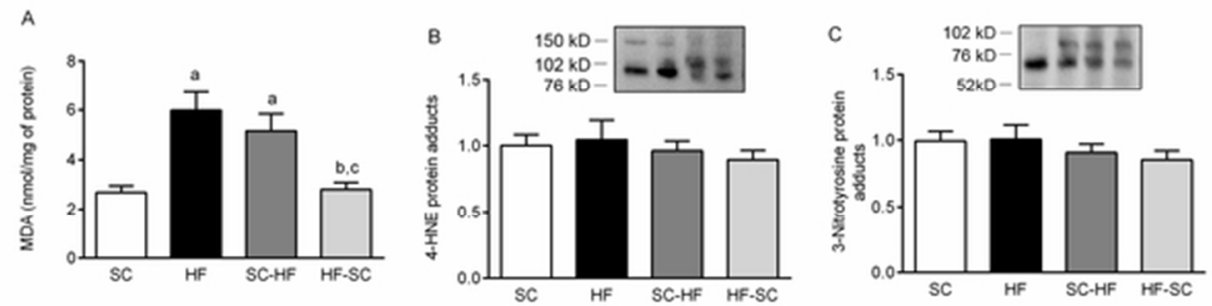

Figure 4. Oxidative stress biomarkers in liver homogenate. (A) malondialdehyde, (B) 4-hydroxynonenal protein adducts, and (C) 3-nitrotyrosine protein adducts. Data are shown as mean \pm SEM. Significant differences are represented by letters $(\mathrm{p} \leq 0.05)$ : [a] vs. SC, [b] vs. HF, [c] vs. SC-HF. HF, high fat; SC, standard chow.

$53 \times 15 \mathrm{~mm}(300 \times 300 \mathrm{DPI})$ 

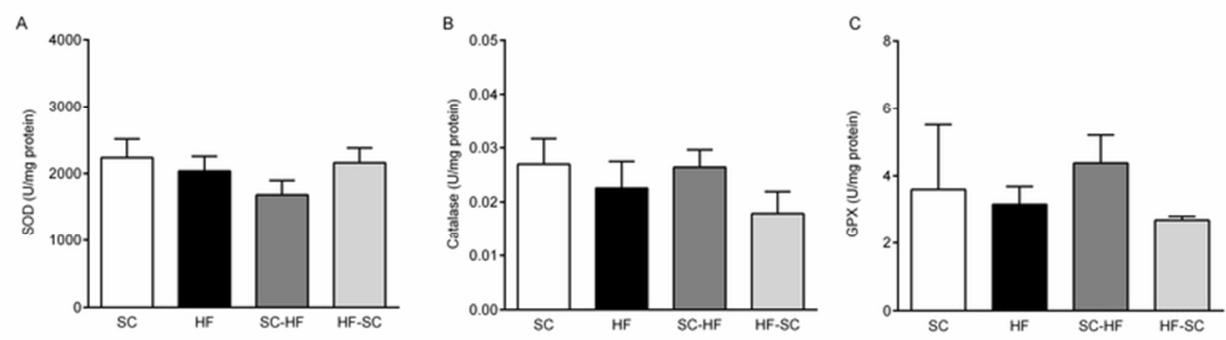

Figure 5. Activity of the antioxidant enzymes (A) superoxide dismutase, (B) catalase, and (C) glutathione peroxidase in liver homogenate. Data are shown as mean \pm SEM. Significant differences are represented by letters $(p \leq 0.05)$ : [a] vs. SC, [b] vs. HF, [c] vs. SC-HF. HF, high fat; SC, standard chow.

$69 \times 20 \mathrm{~mm}(300 \times 300 \mathrm{DPI})$ 


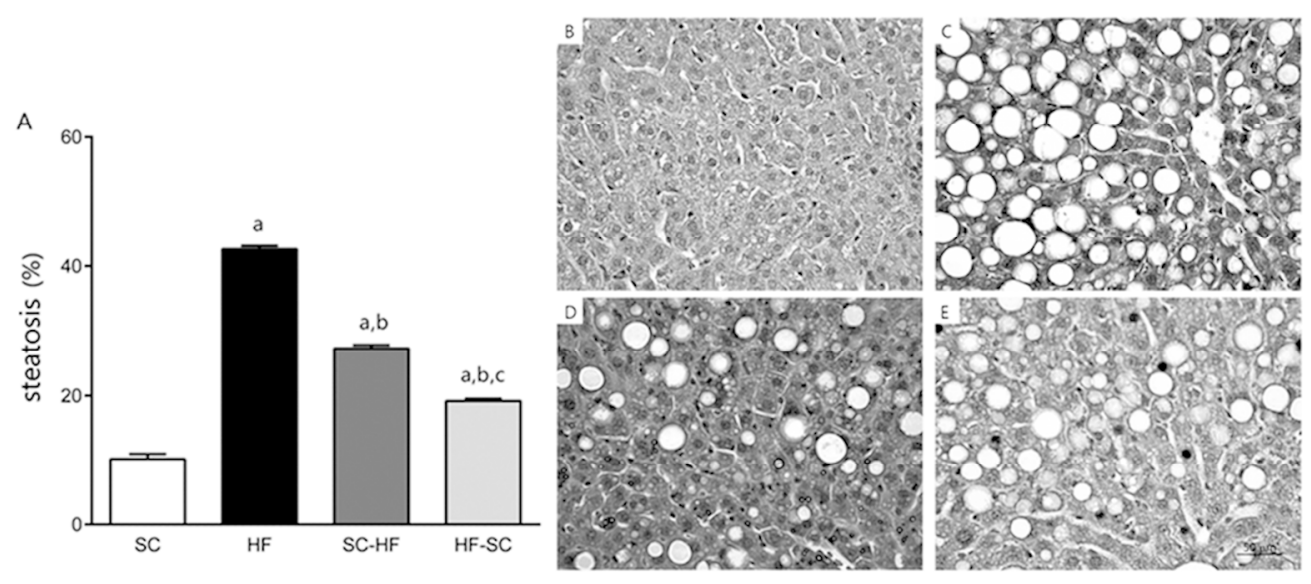

Figure 6. Liver steatosis. (A) bar graph of the volume density of the liver steatosis (mean \pm SEM). Photomicrographs of liver tissue stained with hematoxylin-eosin in (B) SC group, (C), HF group, (D) SC-HF group, and (E) HF-HF group (bar $=50 \mu \mathrm{m}$, same magnification in all photomicrographs). Significant differences are represented by letters $(\mathrm{p} \leq 0.05)$ : [a] vs. SC, [b] vs. HF, [c] vs. SC-HF. HF, high fat; SC, standard chow. 